\title{
Loop near-rings and unique decompositions of $\mathrm{H}$-spaces
}

\author{
DAMIR FRANETIČ \\ PETAR PAVEŠić
}

\begin{abstract}
For every $\mathrm{H}$-space $X$, the set of homotopy classes $[X, X]$ possesses a natural algebraic structure of a loop near-ring. Albeit one cannot say much about general loop near-rings, it turns out that those that arise from $\mathrm{H}$-spaces are sufficiently close to rings to have a viable Krull-Schmidt type decomposition theory, which is then reflected into decomposition results of $\mathrm{H}$-spaces. In the paper, we develop the algebraic theory of local loop near-rings and derive an algebraic characterization of indecomposable and strongly indecomposable $\mathrm{H}$-spaces. As a consequence, we obtain unique decomposition theorems for products of $\mathrm{H}$-spaces. In particular, we are able to treat certain infinite products of $\mathrm{H}$-spaces, thanks to a recent breakthrough in the Krull-Schmidt theory for infinite products. Finally, we show that indecomposable finite $p$-local $\mathrm{H}$-spaces are automatically strongly indecomposable, which leads to an easy alternative proof of classical unique decomposition theorems of Wilkerson and Gray.
\end{abstract}

$55 \mathrm{P} 45 ; 16 \mathrm{Y} 30$

\section{Introduction}

In this paper, we discuss relations between unique decomposition theorems in algebra and homotopy theory. Unique decomposition theorems usually state that sum or product decompositions (depending on the category) whose factors are strongly indecomposable are essentially unique. The standard algebraic example is the KrullSchmidt-Remak-Azumaya theorem. In its modern form, the theorem states that any decomposition of an $R$-module into a direct sum of indecomposable modules is unique, provided that the endomorphism rings of the summands are local rings; see Facchini [8, Theorem 2.12]. Modules with local endomorphism rings are said to be strongly indecomposable, and they play a pivotal role in the study of cancellation and unique decomposition of modules. For example, every indecomposable module of finite length is strongly indecomposable, which implies the classical Krull-Schmidt theorem; see [8, Lemma 2.21 and Corollary 2.23].

Similar results on unique decompositions have been obtained by P Freyd [12] and $\mathrm{H}$ Margolis [18] in the stable homotopy category, and by C Wilkerson [24] and B Gray [14] 
in the unstable homotopy category. However, even when their arguments closely parallel the standard algebraic approach, the above authors choose to rely on specific properties of topological spaces and avoid reference to purely algebraic results. In [21], the second author considered factorizations in the stable homotopy category from the algebraic viewpoint. He first pointed out that the endomorphism rings of stable $p$-complete spectra are finite $\widehat{\mathbb{Z}}_{p}$-algebras, and that those are known to be semiperfect; see Lam [17, Example 23.3]. The unique decomposition for finite $p$-complete spectra then follows immediately because the Krull-Schmidt-Remak-Azumaya theorem holds for modules whose endomorphism ring is semiperfect.

The $p$-local case is more difficult, but Pavešić was able to show (see [21, Theorem 2.1]) that the endomorphism rings of finite $p$-local spectra are also semiperfect rings, which implies that finite $p$-local spectra decompose uniquely. The efficiency of the algebraic approach motivated our attempt to derive unique decomposition theorems in the unstable homotopy category. The leading idea is that the set $[X, X]$ of homotopy classes of selfmaps of $X$ should play a role in the decomposition theory of $\mathrm{H}$-spaces; this is analogous to the role of endomorphism rings in the decomposition of modules. However, the situation is more complicated because of the fact that for a general $\mathrm{H}$-space $X$, the set $[X, X]$ is not a ring but possesses only the much weaker structure of a loop near-ring. Thus we were forced to first develop a notion of localness for loop near-rings, and then to characterize $\mathrm{H}$-spaces that are strongly indecomposable and appear as prime factors in unique decompositions. One of the important advantages of our approach is that there are stronger versions of the Krull-Schmidt-Remak-Azumaya theorem that can be used to derive new decomposition theorems. In particular, a recently proven result about unique decompositions of infinite products of modules led to new unique decomposition theorems for infinite products of $\mathrm{H}$-spaces; see Theorems 2.11 and 2.13 below.

The paper is organized as follows. In Section 1, we study the set of homotopy classes $\operatorname{End}(X):=[X, X]$ for a connected $\mathrm{H}$-space $X$ and show that it has the algebraic structure of a loop near-ring. Since this structure is not well known, we then recall some basic facts about loop near-rings, generalize the concept of localness to loop near-rings and prove the most relevant results. More algebraic details are developed by the first author in [10]. In Section 2, we define strongly indecomposable H-spaces and show that a decomposition of an $\mathrm{H}$-space as a product of strongly indecomposable factors is essentially unique. Finally, in Section 3, we prove that for finite $p$-local $\mathrm{H}$-spaces, indecomposable implies strongly indecomposable, which in turn yields a unique decomposition theorem for $p$-local H-spaces.

Our approach can be almost directly dualized to simply connected coH-spaces and connective CW-spectra. See Remarks 2.2 and 3.4. All spaces under consideration are assumed to be pointed and to have the homotopy type of a connected CW-complex. 
Maps and homotopies are base-point preserving, but we omit the base points from the notation and do not distinguish between a map and its homotopy class.

\section{Loops and loop near-rings}

If $X$ is an $\mathrm{H}$-space, then the set $[X, X]$ of homotopy classes of self-maps admits two natural binary operations. The first, multiplication, is induced by the composition $f g$ of maps $f, g: X \rightarrow X:$ it is associative with the identity map $\mathbf{1}_{X}: X \rightarrow X$ acting as the neutral element, so the resulting algebraic structure $([X, X], \cdot)$ is a monoid. The second operation, addition, is induced by the $\mathrm{H}$-structure: it is neither commutative nor associative in general, and the constant map $\mathbf{0}_{X}: X \rightarrow X$ represents the neutral element. If the H-space $X$ is connected, then $([X, X],+)$ is a so called (algebraic) loop; see Zabrodsky [26, Theorem 1.3.1]. Moreover, addition and composition on $[X, X]$ are related by right distributivity, ie $(f+g) h=f h+g h$ holds for all $f, g, h: X \rightarrow X$. The resulting algebraic structure $\operatorname{End}(X):=([X, X],+, \cdot)$ is called a (right) loop near-ring, a structure that was first introduced by Ramakotaiah and Santhakumari [23]. We are not aware of any papers on loop near-rings that arise in topology. However, if $X$ is an H-group, then $\operatorname{End}(X)$ is a near-ring, and this stronger structure has already been studied by Curjel [7] and, more recently, by Baues, Hartl and Pirashvili [3], and others.

\subsection{Basic properties}

The definition of a loop near-ring is similar to that of a ring, but it lacks some important ingredients: addition is not required to be commutative nor associative, and only one of the distributivity laws is present. The resulting structure is often very different from a ring, but nevertheless, a surprising number of concepts and facts from ring theory can be suitably extended to this more general setting. We recall the definitions and state relevant results.

Definition 1.1 An (algebraic) loop consists of a set $G$ equipped with a binary operation + satisfying the following properties:

- for every $a, b \in G$, the equations $a+x=b$ and $y+a=b$ have unique solutions $x \in G$ and $y \in G$, respectively;

- there exists a two-sided zero, ie an element $0 \in G$ such that $0+a=a+0=a$ for all $a \in G$.

A loop is essentially a "nonassociative group". Existence of unique solutions to equations implies that left and right cancellation laws hold in a loop. We can define the 
operations of left and right difference $\backslash$ and - where $x=a \backslash b$ is the unique solution of the equation $a+x=b$ and $y=b-a$ is the unique solution of the equation $y+a=b$. A loop homomorphism is a function $\phi: G \rightarrow H$ between loops $G$ and $H$ such that $\phi(a+b)=\phi(a)+\phi(b)$ for all $a, b \in G$. Since $\phi(0)=\phi(0)+\phi(0)$, the cancellation in $H$ gives $\phi(0)=0$. Similarly, we get $\phi(a \backslash b)=\phi(a) \backslash \phi(b)$ and $\phi(a-b)=$ $\phi(a)<\phi(b)$.

As in the theory of groups, we can define two kinds of subobjects: subloops and normal subloops. A subset of a loop $G$ is a subloop of $G$ if it is closed with respect to the addition and both difference operations. A direct definition of a normal subloop is more complicated, as we must take into account the nonassociativity of the addition: a subloop $K \leq G$ is a normal subloop if, for all $a, b \in G$, we have

$a+K=K+a, \quad(a+b)+K=a+(b+K) \quad$ and $\quad(K+a)+b=K+(a+b)$.

We often use a slicker characterization: a subset of $G$ is a subloop if it is the image of some loop homomorphism; it is a normal subloop if it is a kernel of some loop homomorphism. See Bruck [4, Chapter IV] for a detailed treatment of these concepts.

Definition 1.2 A (right) loop near-ring $(N,+, \cdot)$ consists of a set $N$ with two binary operations + and $\cdot$ such that:

- $(N,+)$ is a loop,

- $(N, \cdot)$ is a monoid, ie multiplication is associative with identity 1 ,

- multiplication is right distributive over addition, and $n 0=0$ holds for every $n \in N$.

If $(N,+)$ is a group, $(N,+, \cdot)$ is a near-ring.

Our definition of a loop near-ring is made a bit more restrictive than the one given in Ramakotaiah and Santhakumari [23] by requiring that there exists a neutral element for the multiplication, and that $N 0=0$. Both modifications are motivated by the fact that, for an $\mathrm{H}$-space $X$, we have that $\operatorname{End}(X)$ is always unital, and the constant map $0: X \rightarrow X$ satisfies the property $0 n=n 0=0$. In general, right distributivity and cancellation imply $0 n=0$. The symmetric relation $n 0=0$, however, is not automatic and characterizes the so-called zero-symmetric loop near-rings in [23]. Let us also remark that if $X$ is a simply connected coH-space, then $\operatorname{End}(X)$ turns out to be a left loop near-ring.

A generic example of a right near-ring is the near-ring $M(G)$ of all functions $f: G \rightarrow G$ from a group $G$ to itself. Moreover, if $G$ is only a loop, then $M(G)$ is a loop nearring [23, Example 1.2]. The following topological examples are more relevant to our discussion. 
Example 1.3 To present an example of a near-ring whose additive group is not commutative, we first need the following general observation. Given an H-space $X$ with the multiplication map $\mu$ and an arbitrary space $Z$, the sum of maps $f, g: Z \rightarrow X$ is given by the composition $f+g:=\mu(f \times g) \Delta$ as in the diagram

$$
f+g: Z \stackrel{\Delta}{\longrightarrow} Z \times Z \stackrel{f \times g}{\longrightarrow} X \times X \stackrel{\mu}{\longrightarrow} X .
$$

This operation is commutative for all spaces $Z$ if and only if $p_{1}+p_{2}=p_{2}+p_{1}$ holds for the two projections $p_{1}, p_{2}: X \times X \rightarrow X$ in $[X \times X, X]$. Indeed, one can directly check that $f+g=\left(p_{1}+p_{2}\right)(f, g)$, and $g+f=\left(p_{2}+p_{1}\right)(f, g)$, so if $p_{1}+p_{2}=p_{2}+p_{1}$, then $f+g=g+f$ for every $Z$ and every $f, g: Z \rightarrow X$.

A well-known example of an $\mathrm{H}$-structure that is not homotopy commutative is given by the quaternion multiplication on the 3 -sphere $S^{3}$; see James [15]. By the above remark, it follows that $\left[S^{3} \times S^{3}, S^{3}\right]$ is a nonabelian group; hence $\operatorname{End}\left(S^{3} \times S^{3}\right)$ is a (right) near-ring but not a ring.

Example 1.4 Similarly as in the previous example, one can show that given an $\mathrm{H}$ space $X$, the addition on $[Z, X]$ is associative for all spaces $Z$ if and only if the relation $p_{1}+\left(p_{2}+p_{3}\right)=\left(p_{1}+p_{2}\right)+p_{3}$ holds for the three projections in $[X \times X \times X, X]$. The octonion multiplication on the sphere $S^{7}$ is a familiar example of an $\mathrm{H}$-structure that is not homotopy associative (see James [16]), so the addition in $\left[S^{7} \times S^{7} \times S^{7}, S^{7}\right]$ is not associative. We conclude that $\operatorname{End}\left(S^{7} \times S^{7} \times S^{7}\right)$ is not a near-ring but only a (right) loop near-ring.

Example 1.5 Our final example is a left loop near-ring induced by a coH-space structure. Every element $\gamma: S^{6} \rightarrow S^{3}$ of order 3 in the group $\pi_{6}\left(S^{3}\right) \cong \mathbb{Z} / 12$ is a coH-map, therefore its mapping cone $C:=S^{3} \cup_{\gamma} e^{7}$ is a coH-space. Ganea [13, Proposition 4.1] has proven that $C$ does not admit any associative coH-structures, so in particular, the addition induced by the coH-structure in $[C, C \vee C \vee C]$ is not associative. It follows that $\operatorname{End}(C \vee C \vee C)$ is a (left) loop near-ring but not a near-ring.

\subsection{Local loop near-rings}

The crucial ingredient in the proof of the Krull-Schmidt-Remak-Azumaya theorem is the assumption that there is a factorization of the given module as a direct sum of factors whose endomorphism rings are local. In order to extend this approach to factorizations of H-spaces, we need a suitable definition of local loop near-rings. Local near-rings were introduced by Maxson in [19]. We use the characterization [19, Theorem 2.8] to extend this concept to loop near-rings. A subloop $I \leq N$ is said to be an $N$-subloop 
if $N I \subseteq I$. The role of $N$-subloops in the theory of loop near-rings is analogous to that of ideals in rings.

Definition 1.6 A loop near-ring $N$ is local if it has a unique maximal $N$-subloop $J \lesseqgtr N$.

Let $U(N)$ denote the group of units of the loop near-ring $N$, that is to say, the group of invertible elements of the monoid $(N, \cdot)$. A function $\phi: N \rightarrow N^{\prime}$ is a homomorphism of loop near-rings if $\phi(1)=1, \phi(m+n)=\phi(m)+\phi(n)$, and $\phi(m n)=\phi(m) \phi(n)$ hold for all $m, n \in N$. Clearly $\phi(0)=0$, and if $u \in U(N)$, then $\phi(u) \in U\left(N^{\prime}\right)$. A homomorphism is said to be unit-reflecting if the converse holds, ie if $\phi(n) \in U\left(N^{\prime}\right)$ implies $n \in U(N)$. One of the most remarkable properties of loop near-rings that arise in homotopy theory is that they come equipped with a unit-reflecting homomorphism into a ring (namely, with the representation into an endomorphism of homotopy or homology groups, which is unit-reflecting as a consequence of the Whitehead theorem). It is important to observe that the image of such a homomorphism is always a subring of the codomain. The main properties of local loop near-rings are collected in the following theorem. (We denote by $B-A$ the set-theoretic difference of $B$ and $A$, ie the relative complement of $A$ in $B$.)

Theorem 1.7 (i) In a local loop near-ring $N$, the only idempotents are 0 and 1 .

(ii) A loop near-ring $N$ is local if and only if $N-U(N)$ is an $N$-subloop in $N$. Moreover, in this case, $N-U(N)$ is the unique maximal $N$-subloop.

(iii) Let $\phi: N \rightarrow R$ be a nontrivial and unit-reflecting homomorphism from a loop near-ring $N$ to a ring $R$. If $N$ is local, then $\operatorname{im} \phi$ is a local subring of $R$. Conversely, if $R$ is local, then $N$ is a local loop near-ring.

Proof (i) Let $e=e^{2} \in N$ be an idempotent and write an element $n \in N$ as $n=y+n e$. Multiplying this equation by $e$ from the right we get $n e=(y+n e) e=y e+n e$; hence $y e=0$. Denote by $\operatorname{Ann}(e)$ the annihilator of $e$, ie the subset of all $y \in N$ such that $y e=0$. We have just seen that $N=\operatorname{Ann}(e)+N e$. Both subsets, $\operatorname{Ann}(e)$ and $N e$, are $N$-subloops in $N$ (this is immediate for $N e$; for $\operatorname{Ann}(e)$, use the fact that $N$ is zero-symmetric). Similarly as for unital rings, Zorn's lemma implies that every proper $N$-subloop in $N$ is contained in a maximal $N$-subloop, see [19, Lemma 2.7]. Clearly, Ann $(e)$ and $N e$ cannot both be contained in the unique maximal $N$-subloop $J \lesseqgtr N$. Therefore, either $\operatorname{Ann}(e)=N$ or $N e=N$, which means that either $e=0$ or $e=1$.

(ii) Let $N$ be local and let $J \lesseqgtr N$ be the unique maximal $N$-subloop. We claim that every $u \in N-J$ has a left inverse which is also in $N-J$; then it follows automatically that $N-J \subseteq U(N)$. In fact, if $N u \neq N$, then the $N$-subloop $N u$ is contained in $J$; 
hence $u \in J$. Therefore, for $u \in N-J$, we have $N u=N$; in particular, $k u=1$ for some $k \in N$. Observe that $k \in N-J$ as well. In fact, we have the following chain of implications:

$$
\begin{aligned}
(1-u k) u=u-u k u=u-u=0 & \Rightarrow 1-u k \text { is not left invertible } \\
& \Longrightarrow u k \in N-J \\
& \Longrightarrow k \in N-J
\end{aligned}
$$

We conclude $N-J \subseteq U(N)$. The reverse inclusion $U(N) \subseteq N-J$ is obvious; hence $J=N-U(N)$, which is an $N$-subloop.

For the reverse implication, assume that $N-U(N)$ is an $N$-subloop. Since every proper $N$-subloop $I \lesseqgtr N$ is contained in $N-U(N)$, it is clearly the unique maximal $N$-subloop.

(iii) Call a subset $K \subseteq N$ an ideal if $K$ is the kernel of some loop near-ring homomorphism $\psi: N \rightarrow N^{\prime}$. Every ideal $K$ is also an $N$-subloop. If $N$ is local with unique maximal $N$-subloop $J$, then $K \subseteq J$, and the quotient $N / K \cong \operatorname{im} \psi$ has $J / K$ as the unique maximal $(N / K)$-subloop. (Note that the $\psi$-preimage of an $(N / K)$-subloop in $N / K$ is an $N$-subloop in $N$.) So, in particular, im $\phi$ is a local ring.

For the reverse implication, since $\phi$ is unit-reflecting, we have $\phi^{-1}(R-U(R))=$ $N-U(N)$. As $R$ is a local ring, $R-U(R)$ is a left ideal of $R$ by [17, Theorem 19.1]; therefore, its preimage $N-U(N)$ is an $N$-subloop of $N$. Thus by (ii), $N$ is local.

\section{Uniqueness of decompositions of $\mathrm{H}$-spaces}

The classical Krull-Schmidt-Remak-Azumaya theorem says that a factorization of a module as a direct sum of strongly indecomposable modules is essentially unique. In this section, we use the theory of loop near-rings to prove an analogous result for product decompositions of $\mathrm{H}$-spaces.

Given a space $X$, every self map $f: X \rightarrow X$ induces endomorphisms $\pi_{k}(f) \in$ $\operatorname{End}\left(\pi_{k}(X)\right)$ of the homotopy groups of $X$, which can be combined to obtain the following function:

$$
\beta_{X}: \operatorname{End}(X) \rightarrow \prod_{k=1}^{\infty} \operatorname{End}\left(\pi_{k}(X)\right), \quad f \mapsto f_{\sharp}=\left(\pi_{1}(f), \pi_{2}(f), \pi_{3}(f), \ldots\right) .
$$

A loop near-ring homomorphism $\phi: N \rightarrow M$ is idempotent-lifting if for every idempotent of the form $\phi(n) \in M$, there is an idempotent $e \in N$ such that $\phi(e)=\phi(n)$. 
Proposition 2.1 If $X$ is an $H$-space, then $\beta_{X}$ is a unit-reflecting and idempotent-lifting homomorphism from a loop near-ring to a ring.

Proof We already know that $\operatorname{End}(X)$ is a loop near-ring. All homotopy groups of an $\mathrm{H}$-space are abelian, so the $\operatorname{End}\left(\pi_{k}(X)\right)$ are rings; hence the codomain of $\beta_{X}$ is a direct product of rings. Moreover, $\beta_{X}$ is a homomorphism of loop near-rings because $(f+g)_{\#}=f_{\sharp}+g_{\sharp}$ holds for every H-space $X$, while $(f g)_{\#}=f_{\sharp} g_{\sharp}$ by functoriality. To see that $\beta_{X}$ is unit-reflecting, let $f: X \rightarrow X$ be such that the induced homomorphism $\beta_{X}(f)$ is an isomorphism. Then by the Whitehead theorem, $f$ is a homotopy equivalence, ie a unit element of $\operatorname{End}(X)$. Finally, if $\beta_{X}(f)$ is an idempotent, then by [11, Proposition 3.2], there is a decomposition of $X$ into a product of telescopes $\operatorname{Tel}(f) \times \operatorname{Tel}\left(f \backslash \mathbf{1}_{X}\right)$. The first factor in this decomposition determines an idempotent $e: X \rightarrow \operatorname{Tel}(f) \rightarrow X$ in $\operatorname{End}(X)$ such that $\beta_{X}(e)=\beta_{X}(f)$, proving that $\beta_{X}$ is idempotent-lifting.

Remark 2.2 All results of this section are easily dualized to simply connected coHspaces $X$. As in [11], one replaces $\pi_{*}(X)$ with singular homology groups $H_{*}(X)$, and the homomorphism $\beta_{X}$ with the homomorphism

$$
\alpha_{X}: \operatorname{End}(X) \rightarrow \prod_{k=1}^{\infty} \operatorname{End}\left(H_{k}(X)\right), \quad f \mapsto f_{*}=\left(H_{1}(f), H_{2}(f), H_{3}(f), \ldots\right) .
$$

Product and weak product decompositions of $\mathrm{H}$-spaces are replaced by wedge decompositions of coH-spaces; hence Theorems 2.11 and 2.13 below are replaced by one dual theorem. Moreover, if one replaces the coH-space $X$ by a connective $\mathrm{CW}$-spectrum $X$, the dualized argument remains the same. Observe that even though $\operatorname{End}(X)$ is a genuine ring in the case of $\mathrm{CW}$-spectra, its image under $\alpha_{X}$ can be easier to understand.

Every decomposition of an $\mathrm{H}$-space as a product of two noncontractible spaces, $X \simeq$ $Y \times Z$, determines a nontrivial idempotent $e=j p: X \rightarrow Y \hookrightarrow X$ in $\operatorname{End}(X)$, and conversely, every nontrivial idempotent $f \in \operatorname{End}(X)$ gives rise to a nontrivial product decomposition $X \simeq \operatorname{Tel}(f) \times \operatorname{Tel}\left(f \backslash \mathbf{1}_{X}\right)$.

Definition 2.3 An H-space $X$ is indecomposable if $\mathbf{0}_{X}$ and $\mathbf{1}_{X}$ are the only idempotents in $\operatorname{End}(X)$. Moreover, $X$ is strongly indecomposable if $\operatorname{End}(X)$ is a local loop near-ring.

By Theorem 1.7, every strongly indecomposable H-space is indecomposable. The converse is not true: eg $\operatorname{End}\left(S^{1}\right)=\operatorname{End}\left(S^{3}\right)=\operatorname{End}\left(S^{7}\right) \cong \mathbb{Z}$, so $S^{1}, S^{3}$ and $S^{7}$ are indecomposable $\mathrm{H}$-spaces, but they are not strongly indecomposable since the ring 
of integers is not local. The main result of this paper is that the distinction between indecomposable and strongly indecomposable disappears when one considers finite $p$-local spaces.

Example 2.4 In the sense of Baker and May (see [2, Definition 1.1]), a $p$-local $\mathrm{CW}$-complex or spectrum $X$ is called atomic if its first nontrivial homotopy group, say $\pi_{k_{0}}(X)$, is a cyclic $\mathbb{Z}_{(p)}$-module, and a self map $f: X \rightarrow X$ is a homotopy equivalence whenever $f_{\sharp}: \pi_{k_{0}}(X) \rightarrow \pi_{k_{0}}(X)$ is an isomorphism. This notion of atomicity also appeared earlier in the works of Cohen, Moore and Neisendorfer [6, Section 4]. Note that in this case, $\operatorname{End}\left(\pi_{k_{0}}(X)\right)$ is a local ring, and the loop near-ring homomorphism $\pi_{k_{0}}$ : $\operatorname{End}(X) \rightarrow \operatorname{End}\left(\pi_{k_{0}}(X)\right)$ is unit-reflecting. Hence every atomic complex $X$ in this sense is also strongly indecomposable by Theorem 1.7.

In particular, the spectra $B P$ and $B P\langle n\rangle$ are atomic at all primes [2, Examples 5.1 and 5.4], and the suspensions $\Sigma \mathbb{C P}{ }^{\infty}$ and $\Sigma \mathbb{H} \mathrm{P}^{\infty}$ are atomic at the prime 2 by [2, Propositions 4.5 and 5.9]. Moreover, at the prime $p$, there is a decomposition (see McGibbon [20, Proposition 2.2])

$$
\Sigma \mathbb{C P}_{(p)}^{\infty} \simeq W_{1} \vee \cdots \vee W_{p-1},
$$

where the nonzero integral homology groups of $W_{r}$ are $\tilde{H}_{2 k+1}\left(W_{r}\right)=\mathbb{Z}_{(p)}$ for $k \equiv r$ $\bmod (p-1)$. By [2, Proposition 5.9], the suspension spectra $\Sigma^{\infty} W_{r}$ are atomic, and hence, strongly indecomposable by dual reasoning in view of Remark 2.2. The loop near-ring homomorphism $\Sigma^{\infty}: \operatorname{End}\left(W_{r}\right) \rightarrow \operatorname{End}\left(\Sigma^{\infty} W_{r}\right)$ is unit-reflecting, so the coH-spaces $W_{r}$ are also strongly indecomposable. Therefore, the $\vee$-decomposition (1) is unique by the dual of Theorem 2.9 below.

Lemma 2.5 Let $X$ be an $H$-space, and let $f \in \operatorname{End}(X)$ be an idempotent. Then $f=\mathbf{0}_{X}$ if and only if $\beta_{X}(f)=0$.

Proof It is the "if" part that requires a proof. Assume $\beta_{X}(f)=0$, and let $g$ solve the equation $g+f=\mathbf{1}_{X}$ in $\operatorname{End}(X)$. Then $\beta_{X}(g)=1$, so $g$ is a homotopy equivalence by Proposition 2.1. Using right distributivity in $\operatorname{End}(X)$, we obtain $f=(g+f) f=$ $g f+f$. Canceling $f$, we get $g f=\mathbf{0}_{X}$; hence $f=\mathbf{0}_{X}$ since $g$ is a homotopy equivalence.

Lemma 2.5 combined with Theorem 1.7 yields the following detection principle.

Proposition 2.6 Let $X$ be an $H$-space.

(i) $X$ is indecomposable if and only if the ring $\operatorname{im} \beta_{X}$ contains no proper nontrivial idempotents.

(ii) $X$ is strongly indecomposable if and only if the $\operatorname{ring} \operatorname{im} \beta_{X}$ is local. 
Let $X_{i}$ be H-spaces, set $X:=\prod_{i \in I} X_{i}$, and equip $X$ with the $\mathrm{H}$-space structure induced by the $X_{i}$. Then $\operatorname{End}(X)=[X, X]=\prod_{i \in I}\left[X, X_{i}\right]$ as loops. Denote by $e_{i} \in \operatorname{End}(X)$ the idempotent $j_{i} p_{i}: X \rightarrow X_{i} \hookrightarrow X$ corresponding to the factor $X_{i}$. As a loop, $\left[X, X_{i}\right]$ is naturally isomorphic to $e_{i} \operatorname{End}(X)$, the isomorphism being given by $f \mapsto j_{i} f$. Therefore, $\operatorname{End}(X) \cong \prod_{i \in I} e_{i} \operatorname{End}(X)$. Setting $A:=\operatorname{im} \beta_{X}$, it is easily seen that $A=\prod_{i \in I} e_{i \sharp} A$, not only as abelian groups, but also as right $A$-modules. We shall exploit this fact on multiple occasions, as it translates a decomposition problem of an $\mathrm{H}$-space into a (seemingly) more manageable decomposition problem of a module.

Remark 2.7 More can be said. The loop $\left[X, X_{i}\right]$ has a natural right action of the loop near-ring $\operatorname{End}(X)$ given by composition

$$
\left[X, X_{i}\right] \times \operatorname{End}(X) \rightarrow\left[X, X_{i}\right], \quad(f, h) \mapsto f h .
$$

Naturality of the addition on $\left[X, X_{i}\right]$ implies that $(f+g) h=f h+g h$ holds; ie this action is right distributive over + and makes $\left[X, X_{i}\right]$ into an $\operatorname{End}(X)$-comodule (see Clay [5, Definition 13.2]). The isomorphism $\left[X, X_{i}\right] \cong e_{i} \operatorname{End}(X)$ is then an isomorphism of right $\operatorname{End}(X)$-comodules. Of course, once the functor $\pi_{*}$ is applied to $\operatorname{End}(X)=\prod_{i \in I}\left[X, X_{i}\right]$, we obtain the aforementioned identification of right $A-$ modules $A=\prod_{i \in I} e_{i \sharp} A$.

The next technical lemma draws a tight relation between certain retracts of $X$ and corresponding summands of the right $A$-module $A$.

Lemma 2.8 Assume that $Z$ and $Z^{\prime}$ are retracts of an $H$-space $X$, with $Z$ strongly indecomposable. Set $A:=\operatorname{im} \beta_{X}$, and let $e_{\sharp}=(j p)_{\sharp}$ and $e_{\sharp}^{\prime}=\left(j^{\prime} p^{\prime}\right)_{\sharp}$ be the idempotents corresponding to retracts $Z$ and $Z^{\prime}$, respectively. Then $Z$ and $Z^{\prime}$ are homotopy equivalent spaces if and only if $e_{\sharp} A$ and $e_{\sharp}^{\prime} A$ are isomorphic right $A$-modules.

Proof Suppose $Z \simeq Z^{\prime}$. Pick a homotopy equivalence $v: Z \rightarrow Z^{\prime}$ with homotopy inverse $v^{-1}: Z^{\prime} \rightarrow Z$. Consider the elements $\left(j^{\prime} v p\right)_{\sharp}$ and $\left(j v^{-1} p^{\prime}\right)_{\sharp}$ in the ring $A$. Note that $\left(j v^{-1} p^{\prime}\right)_{\sharp}\left(j^{\prime} v p\right)_{\sharp}=e_{\sharp}$ and $\left(j^{\prime} v p\right)_{\sharp}\left(j v^{-1} p^{\prime}\right)_{\sharp}=e_{\sharp}^{\prime}$. For any idempotent $f_{\sharp} \in A$, left multiplication by $f_{\sharp}$ is the identity of the right $A$-module $f_{\sharp} A$. It follows that left multiplication by $\left(j^{\prime} v p\right)_{\sharp}$ is an endomorphism of the right $A$-module $A$, which maps $e_{\sharp} A$ isomorphically onto the submodule $e_{\sharp}^{\prime} A$. Hence $e_{\sharp} A \cong e_{\sharp}^{\prime} A$.

For the reverse implication, observe that $e_{\sharp} A e_{\sharp}$ and $\operatorname{im} \beta_{Z}$ are isomorphic as rings, the latter ring being local by Proposition 2.6. Since $e_{\sharp} A \cong e_{\sharp}^{\prime} A$ as right $A$-modules, the idempotents $e_{\sharp}$ and $e_{\sharp}^{\prime}$ are conjugate in $A$, ie $e_{\sharp}^{\prime}=u_{\sharp}^{-1} e_{\sharp} u_{\sharp}$ for some unit $u_{\sharp} \in U(A)$; see [17, Exercise 21.16]. Now form the composed maps

$$
g=\text { puj }^{\prime}: Z^{\prime} \hookrightarrow X \rightarrow X \rightarrow Z \quad \text { and } \quad h=p^{\prime} u^{-1} j: Z \hookrightarrow X \rightarrow X \rightarrow Z^{\prime},
$$


and verify that $g h$ and $h g$ induce the identity endomorphisms of the respective homotopy groups. Therefore, $Z \simeq Z^{\prime}$.

Finite product decompositions of $\mathrm{H}$-spaces behave nicely, as one is tempted to suspect from the module case.

Theorem 2.9 Assume that an $H$-space $X$ admits a (finite) product decomposition $X \simeq X_{1} \times \cdots \times X_{n}$ into strongly indecomposable factors $X_{i}$.

(i) Any indecomposable retract $Z$ of $X$ is strongly indecomposable. Moreover, there is an index $i$ such that $Z \simeq X_{i}$.

(ii) If $X \simeq X_{1}^{\prime} \times \cdots \times X_{m}^{\prime}$ is any other decomposition of $X$ into indecomposable factors $X_{k}^{\prime}$, then $m=n$, and there is a permutation $\varphi$ such that $X_{i} \simeq X_{\varphi(i)}^{\prime}$ holds for all $i$.

Proof Set $A:=\operatorname{im} \beta_{X}$. A retraction $p: X \rightarrow Z$ and its right inverse $j: Z \hookrightarrow X$ determine an idempotent $f_{\sharp}=(j p)_{\sharp}$ in the ring $A$. We also have idempotents $e_{i \sharp}=\left(j_{i} p_{i}\right)_{\sharp} \in A$ and $e_{k \sharp}^{\prime}=\left(j_{k}^{\prime} p_{k}^{\prime}\right)_{\sharp} \in A$ corresponding to the factors $X_{i}$ and $X_{k}^{\prime}$, respectively. Viewing $A$ as a right $A$-module, we see that (i) $f_{\sharp} A$ is a direct summand of $A$, and (ii) $A$ admits two direct-sum decompositions

$$
A=e_{1 \sharp} A \oplus \cdots \oplus e_{n \sharp} A=e_{1 \sharp}^{\prime} A \oplus \cdots \oplus e_{m \sharp}^{\prime} A .
$$

The theorem will now follow almost directly from its algebraic analogues:

(i) By [8, Lemma 2.11], $f_{\sharp} A$ has a local endomorphism ring. Moreover, $f_{\sharp} A$ is isomorphic to some $e_{i \sharp} A$. Since $\operatorname{End}_{A}\left(f_{\sharp} A\right) \cong f_{\sharp} A f_{\sharp} \cong \operatorname{im} \beta_{Z}$ as rings, $Z$ is strongly indecomposable by Proposition 2.6. Hence, by Lemma 2.8, $Z \simeq X_{i}$.

(ii) By Proposition 2.6, the $A$-modules $e_{i \sharp} A$ are indecomposable with local endomorphism rings, and the $A$-modules $e_{k \sharp}^{\prime} A$ are indecomposable. By the Krull-SchmidtRemak-Azumaya theorem [8, Theorem 2.12] there is a bijection $\varphi:\{1, \ldots, n\} \rightarrow$ $\{1, \ldots, m\}$ such that $e_{i \sharp} A$ and $e_{\varphi(i) \sharp}^{\prime} A$ are isomorphic right $A$-modules. Now use Lemma 2.8 to conclude $X_{i} \simeq X_{\varphi(i)}^{\prime}$ for all $i=1, \ldots, n$.

We will use the proof above as a prototypical example of the use of Lemma 2.8 to deduce the uniqueness of $\mathrm{H}$-space decompositions from the uniqueness of module decompositions. The Krull-Schmidt-Remak-Azumaya theorem for modules, however, is a statement about direct-sum decompositions of modules, and is false for general, ie infinite, direct-product decompositions; see Franetič [9, Example 2.1]. The following proposition is a very special case of [9, Theorem 2.4] that will be used later in this section. 
Proposition 2.10 Let $R$ be a proper subring of the rationals, $A$ an $R$-algebra, and $\left\{M_{i}: i \in I\right\}$ and $\left\{N_{k}: k \in K\right\}$ two countable families of indecomposable $A$-modules, which are finitely generated as $R$-modules. Assume that $\operatorname{End}_{A}\left(M_{i}\right)$ are local rings. If there is an isomorphism $\prod_{i \in I} M_{i} \cong \prod_{k \in K} N_{k}$, then there exists a bijection $\varphi: I \rightarrow K$ such that $M_{i} \cong N_{\varphi(i)}$ as $A$-modules.

Fix a proper subring $R \lessgtr \mathbb{Q}$. A connected H-space $X$ is called $R$-local if $\pi_{*}(X)$ is an $R$-module. A connected $R$-local H-space $X$ is called homotopy-finite if $\pi_{*}(X)$ is finitely generated over $R$, and it is called of finite type if $\pi_{k}(X)$ is finitely generated over $R$ for each $k$.

In [14], B Gray proves a unique decomposition theorem for finite-type H-spaces in the $p$-complete setting; see [14, Corollary 1.4]. For $R$-local finite-type H-spaces, we have the following results (Theorems 2.11 and 2.13).

Theorem 2.11 Let $\left\{X_{i}: i \in I\right\}$ and $\left\{X_{k}^{\prime}: k \in K\right\}$ be two families of $R$-local, homotopy-finite $H$-spaces, with all of the $X_{i}$ strongly indecomposable, and all of the $X_{k}^{\prime}$ indecomposable. Assume that the product $\prod_{i \in I} X_{i}$ is of finite type. If the products $\prod_{i \in I} X_{i}$ and $\prod_{k \in K} X_{k}^{\prime}$ are homotopy equivalent, then there exists a bijection $\varphi: I \rightarrow K$ such that $X_{i} \simeq X_{\varphi(i)}^{\prime}$ for all $i$.

Remark 2.12 More often than not, the products in the above statement will not have the homotopy type of a CW-complex, even though we are assuming that the spaces $X_{i}$ and $X_{k}^{\prime}$ are CW-complexes (or have the homotopy type of a CW-complex).

Proof We set $X:=\prod_{i \in I} X_{i}$ and $A:=\operatorname{im} \beta_{X}$, and we use $e_{i}=j_{i} p_{i}$ and $e_{k}^{\prime}=j_{k}^{\prime} p_{k}^{\prime}$ to denote the idempotents in $\operatorname{End}(X)$ corresponding to the factors of each decomposition. Then $A$ is an $R$-algebra, and the right $A$-module $A$ admits two direct product decompositions

$$
A=\prod_{i \in I} e_{i \sharp} A=\prod_{k \in K} e_{k \sharp}^{\prime} A .
$$

By Proposition 2.6, the $A$-modules $e_{i \sharp} A$ are strongly indecomposable, while the $A$-modules $e_{k \sharp}^{\prime} A$ are indecomposable.

View $e_{i \sharp} A$ as an $R$-submodule of $\operatorname{Hom}_{R}\left(\pi_{*}(X), \pi_{*}\left(X_{i}\right)\right)$ via the monomorphism $e_{i \sharp} f_{\sharp} \mapsto p_{i \sharp} f_{\sharp}$. Since $\pi_{*}\left(X_{i}\right)$ is finitely generated over $R$ and $X$ is of finite type, $\operatorname{Hom}_{R}\left(\pi_{*}(X), \pi_{*}\left(X_{i}\right)\right)$, the $R$-module of graded homomorphisms $\pi_{*}(X) \rightarrow \pi_{*}\left(X_{i}\right)$, is finitely generated. As $R$ is noetherian, each $e_{i \sharp} A$ must also be finitely generated as an $R$-module. Similarly, each $e_{k \sharp}^{\prime} A$ is also finitely generated as an $R$-module. Now, $X$ being of finite type forces both index sets, $I$ and $K$, to be at most countable. Hence all of the assumptions of Proposition 2.10 are satisfied, so there is a bijection $\varphi: I \rightarrow K$ 
such that $e_{i \sharp} A$ and $e_{\varphi(i) \sharp}^{\prime} A$ are isomorphic right $A$-modules. By Lemma 2.8, we must have $X_{i} \simeq X_{\varphi(i)}^{\prime}$ for all $i \in I$.

There is another decomposition of spaces often studied in homotopy category, the weak product. Let $X^{\prime}$ be the set of all points $x=\left(x_{i}\right)_{i \in I} \in \prod_{i \in I} X_{i}$ with all but finitely many of the $x_{i}$ equal to the base point $*_{i} \in X_{i}$. Equip the product $\prod_{i \in I} X_{i}$ with the compactly generated topology, and let $X^{\prime}$ inherit the relative topology. We will (deliberately) use the notation $\bigoplus_{i \in I} X_{i}$ for the space $X^{\prime}$ and call it the weak product of the $X_{i}$. Of course, $X^{\prime}$ can also be viewed as a union (direct limit) of all compactly generated finite products of the $X_{i}$. Hence if all of the $X_{i}$ are $T_{1}$-spaces, there is a natural isomorphism $\pi_{*}\left(\bigoplus_{i \in I} X_{i}\right) \cong \bigoplus_{i \in I} \pi_{*}\left(X_{i}\right)$. Also, if all of the $X_{i}$ are $\mathrm{CW}$-complexes, then the topology on $\bigoplus_{i \in I} X_{i}$ is precisely the $\mathrm{CW}$-topology.

Let $\left\{X_{i}: i \in I\right\}$ be a family of H-spaces with additions $\mu_{i}: X_{i} \times X_{i} \rightarrow X_{i}$. Define a map $\mu^{\prime}: X^{\prime} \times X^{\prime} \rightarrow X^{\prime}$ to be the composite

$$
X^{\prime} \times X^{\prime}=\left(\bigoplus_{i \in I} X_{i}\right) \times\left(\bigoplus_{i \in I} X_{i}\right) \stackrel{\tau}{\longrightarrow} \bigoplus_{i \in I}\left(X_{i} \times X_{i}\right) \stackrel{\oplus_{i \in I} \mu_{i}}{\longrightarrow} \bigoplus_{i \in I} X_{i}=X^{\prime},
$$

where $\tau$ is the coordinate-shuffle map, ie $\tau\left(\left(x_{i}\right)_{i \in I},\left(y_{i}\right)_{i \in I}\right)=\left(x_{i}, y_{i}\right)_{i \in I}$. Clearly, $\tau$ is well defined. Continuity of $\tau$ is assured by equipping all the products above with the compactly generated topology. A routine exercise shows that $\mu^{\prime} j_{1}^{\prime} \simeq \mathbf{1}_{X^{\prime}} \simeq \mu^{\prime} j_{2}^{\prime}$ holds for the two inclusions $j_{1}^{\prime}, j_{2}^{\prime}: X^{\prime} \hookrightarrow X^{\prime} \times X^{\prime}$. (Let $j_{i 1}: X_{i} \hookrightarrow X_{i} \times X_{i}$ be the inclusions of the first factor, and suppose $H_{i}: X_{i} \times \mathbb{I} \rightarrow X_{i}$ are homotopies rel $*_{i}$ from $\mu_{i} j_{i 1}$ to $\mathbf{1}_{X_{i}}$. Consider the composite

$$
H^{\prime}: X^{\prime} \times \mathbb{I} \hookrightarrow\left(\prod_{i \in I} X_{i}\right) \times \mathbb{I} \stackrel{\tau}{\longrightarrow} \prod_{i \in I}\left(X_{i} \times \mathbb{I}\right) \stackrel{\Pi_{i \in I} H_{i}}{\longrightarrow} \prod_{i \in I} X_{i},
$$

where $\mathbb{I} \hookrightarrow \mathbb{I}^{I}$ is the diagonal inclusion. Note that, in fact, $H^{\prime}\left(X^{\prime} \times \mathbb{I}\right) \subseteq X^{\prime}$ since the homotopies $H_{i}$ are rel $*_{i}$. Therefore, $H^{\prime}$ is a homotopy rel $\left(*_{i}\right)_{i \in I}$ from $\mu^{\prime} j_{1}^{\prime}$ to $\mathbf{1}_{X^{\prime}}$. Repeat for $j_{2}^{\prime}$.) Hence, $\bigoplus_{i \in I} X_{i}$ is also an H-space.

Let $X_{i}(i \in I)$ be H-spaces, and let $X^{\prime}=\bigoplus_{i \in I} X_{i}$ be the weak product of the $X_{i}$. Again, we denote by $e_{i}=j_{i} p_{i}$ the idempotent in $\operatorname{End}\left(X^{\prime}\right)$ corresponding to the factor $X_{i}$. The functor $\pi_{*}$ maps $\operatorname{End}\left(X^{\prime}\right)=\left[X^{\prime}, X^{\prime}\right]$ into

$$
\operatorname{End}_{R}\left(\pi_{*}\left(X^{\prime}\right)\right)=\operatorname{Hom}_{R}\left(\bigoplus_{i \in I} \pi_{*}\left(X_{i}\right), \pi_{*}\left(X^{\prime}\right)\right)=\prod_{i \in I} \operatorname{Hom}_{R}\left(\pi_{*}\left(X_{i}\right), \pi_{*}\left(X^{\prime}\right)\right),
$$

and there is a natural identification $\operatorname{Hom}_{R}\left(\pi_{*}\left(X_{i}\right), \pi_{*}\left(X^{\prime}\right)\right) \cong \operatorname{End}_{R}\left(\pi_{*}\left(X^{\prime}\right)\right) e_{i \sharp}$. Set $A^{\prime}:=\operatorname{im} \beta_{X^{\prime}}$. Restricting the decomposition above to the subring $A^{\prime}$ of $\operatorname{End}_{R}\left(\pi_{*}\left(X^{\prime}\right)\right)$, we get $A^{\prime}=\prod_{i \in I} A^{\prime} e_{i \sharp}$ as a left $A^{\prime}$-module. 
We can now state the weak product version of Theorem 2.11. The proof is deliberately omitted, as it uses the same argument as the proof of Theorem 2.11 with the left $A^{\prime}$-module $A^{\prime}$ in place of the right $A$-module $A$.

Theorem 2.13 Let $\left\{X_{i}: i \in I\right\}$ and $\left\{X_{k}^{\prime}: k \in K\right\}$ be two families of $R$-local, homotopy-finite $H$-spaces, with all of the $X_{i}$ strongly indecomposable and all of the $X_{k}^{\prime}$ indecomposable. Assume that the weak product $\bigoplus_{i \in I} X_{i}$ is of finite type. If the weak products $\bigoplus_{i \in I} X_{i}$ and $\bigoplus_{k \in K} X_{k}^{\prime}$ are homotopy equivalent, then there exists a bijection $\varphi: I \rightarrow K$ such that $X_{i} \simeq X_{\varphi(i)}^{\prime}$ for all $i$.

Of course, the above uniqueness theorems say nothing about the existence of factorizations of $\mathrm{H}$-spaces as products of strongly indecomposable spaces. For example, if $X$ is an $\mathrm{H}$-space having the homotopy type of a finite $\mathrm{CW}$-complex, then one can decompose $X$ as a product of indecomposable factors, but these factors will rarely be strongly indecomposable (unless $\operatorname{End}(X)$ is finite). This is reflected in the well-known phenomenon that finite $\mathrm{H}$-spaces often admit inequivalent product decompositions. The situation becomes more favorable if we consider $p$-localizations of H-spaces. In the following section, we are going to show that a $p$-local finite $\mathrm{H}$-space is indecomposable if and only if it is strongly indecomposable. A factorization of an $\mathrm{H}$-space as a product of such spaces is therefore unique. Finally, if we consider $p$-complete H-spaces, then even the finite-dimensionality assumption may be dropped. In fact, Adams and Kuhn [1] have proven that every indecomposable $p$-complete $\mathrm{H}$-space of finite type is atomic, which implies in particular that each such $\mathrm{H}$-space is strongly indecomposable. Theorem 2.9 implies that decompositions into finite products of $p$-complete atomic spaces are unique. For an alternative approach that works for spaces of finite type, see Gray [14, Corollaries 1.4 and 1.5] or Xu [25, Theorem 4.2.14].

\section{Homotopy endomorphisms of $p$-local spaces}

In this section, we consider $p$-local $\mathrm{H}$-spaces and show that under suitable finiteness assumptions, the indecomposability of a space implies strong indecomposability. The proof is an interesting blend of topology and algebra since it uses nontrivial results from homotopy theory, the theory of local rings and the theory of loop near-rings. Let us say that, for some subring $R \leq \mathbb{Q}$, an $R$-local H-space $X$ is finite if it is finite-dimensional and if its homotopy groups are finitely generated $R$-modules. For every finite $\mathrm{H}$-space $X$, we can define the homomorphism

$$
\bar{\beta}_{X}: \operatorname{End}(X) \rightarrow \prod_{k=1}^{\operatorname{dim} X} \operatorname{End}\left(\pi_{k}(X)\right), \quad f \mapsto\left(\pi_{1}(f), \pi_{2}(f), \ldots, \pi_{\operatorname{dim} X}(f)\right) .
$$


We will show that, when applied to a finite H-space $X$, the homomorphism $\bar{\beta}_{X}$ retains the same main features of the homomorphism $\beta_{X}$ as described in Proposition 2.1, but it has a great advantage over the latter because it maps into the ring of endomorphisms of a finitely generated module.

Proposition 3.1 If $X$ is a finite $H$-space, then the homomorphism $\bar{\beta}_{X}$ is unit-reflecting and idempotent-lifting.

Proof Reflection of units follows from the Whitehead theorem, so it only remains to prove that $\bar{\beta}_{X}$ is idempotent lifting.

First observe that finite $\mathrm{H}$-spaces are rationally elliptic; ie $X$ is rationally equivalent to a finite product of Eilenberg-MacLane spaces: $X_{\mathbb{Q}} \simeq K\left(\mathbb{Q}, n_{1}\right) \times \cdots \times K\left(\mathbb{Q}, n_{t}\right)$; see [26, Section 4.4]. It follows that for all $k>\operatorname{dim} X$, the groups $\pi_{k}(X)$ are torsion and, hence, finite.

Let a map $f: X \rightarrow X$ be such that $\pi_{k}(f)=\pi_{k}(f)^{2}: \pi_{k}(X) \rightarrow \pi_{k}(X)$ for all $k \leq \operatorname{dim} X$; ie $\bar{\beta}_{X}(f)$ is an idempotent in $\operatorname{im} \bar{\beta}_{X}$. As the groups $\pi_{k}(X)$ are finite for $k>\operatorname{dim} X$, there is an integer $n$ such that the $n$-fold composite $f^{n}: X \rightarrow X$ induces an idempotent endomorphism $\pi_{k}(f)^{n}: \pi_{k}(X) \rightarrow \pi_{k}(X)$ for all $k \leq 2(\operatorname{dim} X+1)$. If we set $\bar{f}:=f^{n} \backslash \mathbf{1}_{X}$ in the loop near-ring $\operatorname{End}(X)$, then $\pi_{k}(\bar{f})=\mathbf{1}_{\pi_{k}(X)}-\pi_{k}(f)^{n}$ is an idempotent endomorphism of $\pi_{k}(X)$ for all $k \leq 2(\operatorname{dim} X+1)$. It follows that the map

$$
X \stackrel{\Delta}{\rightarrow} X \times X \hookrightarrow \operatorname{Tel}\left(f^{n}\right) \times \operatorname{Tel}(\bar{f})
$$

induces an isomorphism

$$
\pi_{k}(X) \rightarrow \operatorname{im} \pi_{k}(f)^{n} \oplus \operatorname{im} \pi_{k}(\bar{f})
$$

for all $k \leq 2(\operatorname{dim} X+1)=\operatorname{dim}\left(\operatorname{Tel}\left(f^{n}\right) \times \operatorname{Tel}(\bar{f})\right)$. Hence, $X \simeq \operatorname{Tel}\left(f^{n}\right) \times \operatorname{Tel}(\bar{f})$ by the Whitehead theorem. This product decomposition determines the idempotent $e: X \rightarrow$ $\operatorname{Tel}\left(f^{n}\right) \rightarrow X$ that satisfies $\pi_{k}(e)=\pi_{k}(f)^{n}=\pi_{k}(f)$ for all $k \leq \operatorname{dim} X$. In other words, $\bar{\beta}_{X}(e)=\bar{\beta}_{X}(f)$; therefore, $e$ is an idempotent in $\operatorname{End}(X)$ that lifts $\bar{\beta}_{X}(f)$.

We have now prepared all the ingredients needed for the proof of the main result of this section.

Theorem 3.2 Indecomposable finite $p$-local $H$-spaces are strongly indecomposable.

Proof To simplify the notation, let us denote by $E$ the $\operatorname{ring} \prod_{k=1}^{\operatorname{dim} X} \operatorname{End}\left(\pi_{k}(X)\right)$, by $A$ its subring $\operatorname{im} \bar{\beta}_{X}$, and by $J=J(A)$ the Jacobson radical of $A$.

Theorem 1.7 says that in order to prove that $\operatorname{End}(X)$ is a local loop near-ring, it is sufficient to show that $A$ is a local ring. The ring $A$ is finitely generated as a $\mathbb{Z}_{(p)}-$ module, 
so by [17, Proposition 20.6], the quotient $A / J$ is semisimple (ie a product of full-matrix rings over division rings). Therefore, we must prove that $A / J$ has only trivial idempotents, as this would imply that $A / J$ is a division ring, and hence that $A$ is local. In fact, it is sufficient to prove that $J$ is an idempotent-lifting ideal, because then every nontrivial idempotent in $A / J$ would lift to a nontrivial idempotent in $A$, and then along $\bar{\beta}_{X}$ to a nontrivial idempotent in $\operatorname{End}(X)$, contradicting the indecomposability of $X$.

That $J$ is idempotent-lifting is proved by the following argument. The ring $E$ is semiperfect by [17, Examples 23.2 and 23.4] because it is a product of endomorphism rings of finitely generated $\mathbb{Z}_{(p)}$-modules. By [11, Lemma 3.2], $A$ is a subring of finite additive index in $E$, and so by [22, Example 3.3], the radical $J$ is idempotent-lifting, which concludes the proof.

Let us remark that if $X$ is a $p$-local $\mathrm{H}$-space whose graded homotopy group is a finitely generated $\mathbb{Z}_{(p)}$-module (ie $X$ is a homotopy-finite $p$-local H-space), then the above proof works with $\beta_{X}$ in place of $\bar{\beta}_{X}$, and we obtain the following result as well.

Theorem 3.3 Let $X$ be a $p$-local $H$-space such that its graded homotopy group is a finitely generated $\mathbb{Z}_{(p)}$-module. Then $X$ is indecomposable if and only if it is strongly indecomposable.

Remark 3.4 If $X$ is a simply connected $p$-local coH-space (or a $p$-local connective $\mathrm{CW}$-spectrum), there is no distinction between finite and homology finite (at least up to homotopy equivalence). Theorems 3.2 and 3.3 are therefore replaced by one dual theorem. In the proof of Theorem 3.2 we simply replace the homomorphism $\bar{\beta}_{X}$ with $\alpha_{X}$ without any additional complications. No dual of Proposition 3.1 is needed.

Observe that the two versions of the theorem of Wilkerson [24] on the unique factorization of $p$-local $\mathrm{H}$-spaces now follow as easy corollaries. In fact, every $p$-local $\mathrm{H}$-space of finite type that is either finite-dimensional or homotopy finite-dimensional admits a decomposition as a product of indecomposable factors. By Theorems 3.2 and 3.3, the factors are indeed strongly indecomposable, so by Theorem 2.9, the decomposition is unique.

Example 3.5 One might wonder whether the Theorems 3.2 and 3.3 remain true if we replace finite by finite type (ie $\pi_{k}(X)$ are finitely generated for all $k$ ). We know that at least in the case of CW-spectra, they are false. Consider the example given by Adams and Kuhn in [1, Section 4]. They construct an indecomposable $p$-local spectrum $X$ such that the ring homomorphism $H_{0}: \operatorname{End}(X) \rightarrow \operatorname{End}\left(H_{0}(X)\right)$ is unit-reflecting, and its image is a ring isomorphic to

$$
\frac{\mathbb{Z}_{(p)}[\lambda]}{\left(\lambda^{2}-\lambda+p\right)}
$$


The spectrum $X$ has $H_{0}(X)=\mathbb{Z}_{(p)} \oplus \mathbb{Z}_{(p)}$, so we can identify $\operatorname{End}\left(H_{0}(X)\right)$ with $M_{2}\left(\mathbb{Z}_{(p)}\right)$, the ring of $2 \times 2$ matrices with entries in $\mathbb{Z}_{(p)}$. The image of $\operatorname{End}(X)$ in this matrix ring is precisely the one-to-one image of $\mathbb{Z}_{(p)}[\lambda] /\left(\lambda^{2}-\lambda+p\right)$ under the ring homomorphism which maps a polynomial $q$ to the matrix $q(A)$, where

$$
A=\left(\begin{array}{rr}
0 & 1 \\
-p & 1
\end{array}\right) \text {. }
$$

(Note that $\lambda^{2}-\lambda+p$ is the minimal polynomial of $A$.) Now $A$ is not invertible, and neither is $I-A$, so the ring $\mathbb{Z}_{(p)}[\lambda] /\left(\lambda^{2}-\lambda+p\right)$ cannot be local. Hence $X$ is an indecomposable $p$-local spectrum of finite type, which is not strongly indecomposable.

Adams' and Kuhn's construction of the spectrum $X$ relies on the existence of certain elements in the stable homotopy groups of spheres (in the image of the $J$-homomorphism) and cannot be directly applied to spaces. It remains an open question whether a similar example exists in the realm of finite type $\mathrm{H}$ - or coH-spaces.

Acknowledgement Pavešić was partly supported by the Slovenian Research Agency grant P1-0292-0101, project No. J1-6721-0101.

\section{References}

[1] J F Adams, N J Kuhn, Atomic spaces and spectra, Proc. Edinburgh Math. Soc. 32 (1989) 473-481 MR

[2] A J Baker, J P May, Minimal atomic complexes, Topology 43 (2004) 645-665 MR

[3] H J Baues, M Hartl, T Pirashvili, Quadratic categories and square rings, J. Pure Appl. Algebra 122 (1997) 1-40 MR

[4] R H Bruck, A survey of binary systems, Ergeb. Math. Grenzgeb. 20, Springer, New York (1958) MR

[5] J R Clay, Nearrings: geneses and applications, Clarendon, New York (1992) MR

[6] F R Cohen, J C Moore, J A Neisendorfer, Exponents in homotopy theory, from "Algebraic topology and algebraic $K$-theory" (W Browder, editor), Ann. of Math. Stud. 113, Princeton Univ. Press (1987) 3-34 MR

[7] C R Curjel, On the homology decomposition of polyhedra, Illinois J. Math. 7 (1963) 121-136 MR

[8] A Facchini, Module theory, Progress in Math. 167, Birkhäuser, Basel (1998) MR

[9] D Franetič, A Krull-Schmidt theorem for infinite products of modules, J. Algebra 407 (2014) 307-315 MR

[10] D Franetič, Local loop near-rings, to appear in Rend. Semin. Mat. Univ. Padova (2015) arXiv 
[11] D Franetič, P Pavešić, H-spaces, semiperfect rings and self-homotopy equivalences, Proc. Roy. Soc. Edinburgh Sect. A 141 (2011) 1263-1277 MR

[12] P Freyd, Stable homotopy, from "Proc. Conf. Categorical Algebra" (S Eilenberg, D K Harrison, S Mac Lane, H Röhrl, editors), Springer, New York (1966) 121-172 MR

[13] T Ganea, Cogroups and suspensions, Invent. Math. 9 (1969/1970) 185-197 MR

[14] B Gray, On decompositions in homotopy theory, Trans. Amer. Math. Soc. 358 (2006) 3305-3328 MR

[15] IM James, Multiplication on spheres, I, Proc. Amer. Math. Soc. 8 (1957) 192-196 MR

[16] I M James, Multiplication on spheres, II, Trans. Amer. Math. Soc. 84 (1957) 545-558 MR

[17] T Y Lam, A first course in noncommutative rings, Graduate Texts in Mathematics 131, Springer, New York (1991) MR

[18] H R Margolis, Spectra and the Steenrod algebra, North-Holland Mathematical Library 29, North-Holland, Amsterdam (1983) MR

[19] C J Maxson, On local near-rings, Math. Z. 106 (1968) 197-205 MR

[20] C A McGibbon, Stable properties of rank-1 loop structures, Topology 20 (1981) 109-118 MR

[21] P Pavešić, Endomorphism rings of p-local finite spectra are semi-perfect, Proc. Roy. Soc. Edinburgh Sect. A 139 (2009) 567-574 MR

[22] P Pavešić, Induced liftings, exchange rings and semi-perfect algebras, J. Pure Appl. Algebra 214 (2010) 1901-1906 MR

[23] D Ramakotaiah, C Santhakumari, On loop near-rings, Bull. Austral. Math. Soc. 19 (1978) 417-435 MR

[24] C Wilkerson, Genus and cancellation, Topology 14 (1975) 29-36 MR

[25] K Xu, Algebraic topology: endomorphisms of complete spaces, $\mathrm{PhD}$ thesis, University of Aberdeen (1991)

[26] A Zabrodsky, Hopf spaces, North-Holland Mathematics Studies 22, North-Holland Publishing Co., Amsterdam (1976) MR

Faculty of Computer and Information Science, University of Ljubljana,

Večna pot 113, 1000 Ljubljana, Slovenia

Faculty of Mathematics and Physics, University of Ljubljana,

Jadranska 19, 1111 Ljubljana, Slovenia

damir.franetic@fri.uni-lj.si, petar.pavesic@fmf.uni-lj.si

http://www.fri.uni-lj.si/si/damir-franetic,

http://www.fmf .uni-lj.si/ pavesic

Received: 25 November $2015 \quad$ Revised: 28 February 2016 